\title{
Development of gaze aversion: qualitiative changes over the early school years
}

Gwyneth Doherty-Sneddon, Fiona Phelps and Julia Clarke

\author{
${ }^{1}$ Department of Psychology, University of Stirling \\ ${ }^{2}$ Department of Psychology, University of Cardiff \\ ${ }^{3}$ Department of Psychology, University of Stirling
}

*Requests for reprints should be addressed to Gwyneth Doherty-Sneddon, Department of Psychology, University of Stirling, FK9 4LA, Scotland (e-mail: gds1@stir.ac.uk).

Acknowledgements: This work was supported by an ESRC grant (R000239930) held by Gwyneth

Doherty-Sneddon, Department of Psychology, University of Stirling. We thank the children who participated in this research, and their teachers and parents for their cooperation and consent. 


\begin{abstract}
Looking away from an interlocutors' face during demanding cognitive activity can help adults and children answer challenging mental arithmetic and verbal-reasoning questions (Glenberg, Schroeder, \& Robertson, 1998; Phelps, Doherty-Sneddon \& Warnock, in press). Whilst such 'gaze aversion' (GA) is used far less by 5 -year old school children, its use increases dramatically during the first years of primary education, reaching adult levels by 8 -years of age (Doherty-Sneddon, Bruce, Bonner, Longbotham, \& Doyle, 2002). The current study investigates whether developmental changes also occur in a qualitative aspect of GA - the direction of movement involved in GA shifts. Video data from 18 5-year-olds and 19 8-year-olds answering verbal and arithmetic questions were analysed for direction of GA. We found very different profiles of direction of GA across the two ages: whilst the 5-year-olds used predominantly rapid multi-directional 'flicking' movements and some sustained left lateral movements, the 8year-olds used predominantly sustained rightward movements. It is concluded that, as well as quantitative increases in the use of GA across these age groups, there are concomitant qualitative changes in the nature of GA shifts. A model of human attention in face-to-face interaction is discussed as are implications for the assessment of children's learning and development.
\end{abstract}


During difficult cognitive activity (e.g. remembering information, thinking of an answer to a question, speechplanning, speaking) we often close our eyes, look up at the sky, or look away from the person we are in conversation with (Glenberg et al., 1998; Doherty-Sneddon et al., 2002). Several studies report ways in which adults 'switch off' from environmental stimulation (both live faces and other sorts of visual displays) by averting their gaze, in order to concentrate on cognitive tasks (e.g. Beattie, 1981; Day, 1964; Glenberg et al., 1998) - the cognitive load explanation of GA. Previc, Declerck and Brabander (2005) propose that such gaze shifts during thinking reflect the particular mental activity involved in a task and that this GA is related to the difficulty of active thought processes. Indeed, adults' use of GA has been shown to increase in response to increasingly effortful cognitive activity (e.g. Glenberg et al., 1998). In the forensic field Vrij (2002) reports that GA is a 'nervous behaviour' relating to deception (cf. Zuckerman \& Driver, 1985). When people are intentionally deceiving they feel nervous about being found out and also have to 'think hard' to make their deception convincing. Eight-year old children have also been shown to look away more when answering difficult questions compared with easy ones when questioned face-to-face (Doherty-Sneddon \& Phelps, 2005; Doherty-Sneddon et al., 2002). GA is therefore a potentially useful cue during pedagogical interactions, since it gives a non-verbal indication of a child's level of understanding and concentration (see Doherty-Sneddon et al., 2002).

Additionally, it has been shown that increasing the amount that people avert their gaze improves questionanswering performance (Glenberg et al., 1998; Phelps, Doherty-Sneddon \& Warnock, 2006), suggesting that it serves an important role in problem solving. For example Phelps et al. (2006) trained 5-year-old children to increase the proportion of time they spent averting their gaze during questioning and found that the accuracy of responses to questions increased significantly as a consequence of this training. So, GA may be a useful indicator that attention has been shifted from external sources of information to allow internal reflection upon a question's answer, with the problem's level of difficulty measurable by the amount of GA displayed by a participant. This has important educational implications, especially given the finding, discussed below, that the amount of GA that children engage in during problem solving changes in important ways over the early primary school years (Doherty-Sneddon, 2004; Doherty-Sneddon et al., 2002; Phelps et al., in press).

The theoretical framework that we propose is that visual communication cues are informative and hence carry a processing cost that can interfere with task relevant processing resources. Furthermore the study of GA provides a 
new way of looking not only at human communication but also human attention and thinking. Traditionally human selective attention has been seen in terms of individualistic selective attention whereby we explicity attend to one thing at a time whether this be internal, for example remembering an event, or attention directed to external events (e.g. Posner \& Rothbart 2000). We propose to develop this model as follows. During face-to-face conversation with another person we construct a joint or 'interactional processing space' (IPS)- a constructionist account of on-line thinking. The verbal and non-verbal messages from both parties contribute to IPS with the cognitive processing of all involved driven at any given moment by the person holding the conversational floor. When an individual has to rely on their own internal processing, for example when they have to retrieve a piece of information from their own memory, they have to 'switch off' from the IPS and give executive control to their own executor again. Gaze aversion away from an interlocutor indicates that the averter is switching attentional resources from the IPS to internal or individualistic processing.

Empirical work suggests that children start to use GA whilst thinking (and, to a lesser extent, speaking) from around 5 years of age (e.g. Doherty-Sneddon et al., 2002; Phelps et al., in press). Indeed, it has been argued that a significant developmental surge in the use of GA behaviours during thought occurs between 5 and 6 years of age (Phelps et al., in press); a behaviour which continues to develop (albeit less markedly) throughout the proceeding two years of age. So, by the time children have reached 8 years of age, children use GA like adults to help them manage cognitive load (Doherty-Sneddon \& Phelps, 2005; Doherty-Sneddon et al., 2002). In contrast, 5-year-old children have been shown to use GA to a much lesser extent, and also fail to consistently increase their use of GA in response to increasingly difficult questions although some evidence for this does occur (Doherty-Sneddon et al., 2002; Phelps et al., in press).

These earlier studies which report developmental trajectories in the use of GA during the early primary years (Doherty-Sneddon et al., 2002; Phelps et al., in press) involved looking at the percentage of time children spent using GA whilst thinking about the answers to increasingly difficult mental arithmetic and verbal reasoning questions. However, the current study measures a different, qualitative aspect of the development of GA; namely the direction of GA that children of different ages use while thinking about verbal and mathematical problems. This has important developmental and theoretical implications regarding the factors that determine why children (and 
indeed adults) avert their gaze during questioning. For example, is direction of GA related to cognitive function in the same way for younger and older children?

There is of course existing evidence in the literature showing that children's attention shifting abilities change dramatically over the early primary school years (e.g. Pearson \& Lane, 1991). GA may therefore be an attention shifting strategy that develops significantly between the ages of 5 and 8 years. In the current study we predict that GA will change qualitatively during this time reflecting more sophisticated attention shifting mechanisms. If GA in response to cognitive difficulty is even partially under voluntary control and reflects an executively driven attention shifting strategy, involvement of the prefrontal cortex would be predicted (Banich, 2004) as would hemispheric lateralisation of neurological activity depending upon the nature of concurrent cognitive activity - see below for a discussion. In contrast lower level attention shifting is associated with a number of precortical structures, for example the anterior cingulate cortex, which is also associated with increasing task demands, (Paus Koski, Caramanos, \& Westbury 1998). In addition the superior colliculus (SC) is known to be involved in the generation of saccadic eye movements and eye-head coordination. The SC is connected to many sensori-motor areas of the brain, receives visual and auditory inputs and is thought to help orient the head and eyes toward something seen and heard; the SC clearly provides a candidate precortical region for a specific attention shifting mechanism associated with gaze aversion. In this study we investigate whether there are different patterns of GA movement in different age groups. If younger children's GA reflects precortical attention shifting we would expect saccade-like (rapid), less lateralised movements. In contrast prefrontal cortex, executively controlled attention shifting will allow suppression of saccadic movements allowing more sustained GA.

A related issue is whether particular directions of GA are favoured over others. Previous research has examined the relationship between hemispheric activation (a physiological measure of cognitive activity) and direction of GA, leading to the conclusion that direction of GA during cognitive activity is contralaterally controlled. Kinsbourne (1972) proposed that when a left hemisphere task (e.g. verbal question) is addressed, stimulation of the left hemisphere is argued to connect to the right frontal eye field influencing the saccadic system, resulting in a rightward lateral eye movement (LEM). Conversely, a right hemisphere task (e.g. spatial question) is argued to result in a leftward LEM (see also Griffiths \& Woodman, 1985). Similarly Galluscio and Paradzinski (1995) found more leftward eye movements when adults performed spatial tasks and more rightward and upward movements 
when verbal tasks were engaged in. Analysis of their participants' performance accuracies revealed the verbal task to be more difficult than the spatial which may lead to the conclusion that harder tasks were more associated with upward movements.

However the contralateral pattern of GA is not always found and indeed the literature is full of contradictory findings. In contrast to the view that GA is contralaterally controlled, it has been argued that the direction of LEMs is subject to individual differences between participants. According to this perspective, individuals can be said to have a dominant direction in which they avert their gaze; a direction which remains constant irrespective of the type (i.e. spatial or verbal) of cognitive activity being engaged in. In support of this view there is some evidence that LEMs can be consistently in a given direction for a given person for both left- and right-hemisphere tasks, suggesting that people are either 'left-movers' or 'right-movers' (Day, 1964; Duke, 1968; Bakan, 1969). Related to this is the proposal by Gur, Gur and Harris (1975) that social factors such as performance anxiety in face-to-face interaction may lead individuals to rely on their dominant hemisphere during task processing.

An alternative, but related position which also sees GA as a 'by-product' of cognitive processing is taken by Previc et al (2005) and Previc and Murphy (1997), who studied 11-year-olds and adults respectively, and found a high prevalence of upward eye movements in response to a variety of types of questions. They proposed that this preponderance of upward LEMs reflects engagement in demanding cognitive activity and shares common underlying neural machinery with higher cognition (e.g. abstract thought). Accordingly, Previc et al. argue that there exists a link between upward LEMs and thought because both make use of saccadic neural machinery in the lateral prefrontal cortex and other oculomotor association cortical regions; regions which are of relevance to higher human thought because of their orientation to distant space (Pierrot-Deseilligny, Muri, Ploner, Gaymard, \& Rivaud-Pechoux, 2003) and association with working memory (Kane \& Engle, 2002).

The current study makes an important contribution to existing theories linking GA with cognitive processing. Two accounts discussed above, the contralaterally-influenced LEM work and the Previc et al (2005) account see GA as a by-product of underlying processing. In contrast the cognitive load hypothesis (Glenberg et al 1998; DohertySneddon et al 2002) sees GA as a behavioural 'strategy' to influence efficiency of processing. Of course these divergent approaches may indeed be consonant with one another - directional GA may reflect the nature of the 
cognitive activity that it benefits. It is therefore important that we study not only the development of the occurrence of GA per se in relation to cognitive difficulty (Glenberg et al 1998; Doherty-Sneddon et al 2002; DohertySneddon et al 2005) but also understand the qualitative development of patterns of GA. This of course also has implications for practical applications. For example will the types of GA movements witnessed by teachers when children are engaged in demanding mental activities differ depending on the age of the child?

In the current paper we look at 5- and 8-year-old children's direction of GA movements whilst answering arithmetic and verbal reasoning questions in a face-to-face context; questions which are both associated with left hemisphere function (Buchel, 2003; Delazer, Domahs, Bartha, Brenneis, Lochy, Trieb, \& Benke, 2003). Mental arithmetic and verbal reasoning questions were used in an attempt to mimic the type of cognitive activity required of both 5- and 8-year-old children in everyday classroom situations; a context whereby face-to-face interactions predominate. In addition, these represent the types of questions used in existing studies which have measured proportion of time of GA during thought (e.g. Doherty-Sneddon et al., 2002; Glenberg et al., 1998; Phelps et al., in press). The questions we address are as follows: first, do age-related differences exist in the directional and temporal patterns of GA movements during face-to-face questioning? Second, will our two left hemispheric tasks (mental arithmetic and verbal reasoning) result in predominantly rightward GA movements as the contralaterallyinfluenced LEM account would predict? Third, does cognitive difficulty influence GA direction, for example more upward movements during difficult questions (Previc et al, 2005)? Other secondary hypothesis are also tested by the analyses reported. Whilst there is a substantial literature investigating adult LEMs there is a dearth in relation to how this important aspect of eye gaze develops in childhood. So, we know that the quantity of GA changes dramatically during these years, studying the qualitative changes in the nature of GA will help us extend our understanding of the origins of this important behaviour during social interaction and will inform the application of this knowledge. The current study re-analyses data previously collected for two published studies (DohertySneddon \& Phelps, 2005; Phelps et al, 2006). The 8-year-old children's data is from Doherty-Sneddon \& Phelps (2005). As part of the original study the 8-year-old children also answered autobiographical memory and episodic memory questions as well as questions of moderate difficulty. In the current study only the data from verbal reasoning and mental arithmetic questions that were either at an easy or hard level of difficulty were subject to reanalysis along with similar data for the 5-year-olds taken from Phelps et al (2006). 
Method

\section{Participants}

Nineteen 8 -year-old children $(9$ boys and 10 girls; $M$ age $=8$ years 4 months, range $=8$ years 3 month to 9 years 5 months) and 18 -year-old children (4 boys and 14 girls; $M$ age $=5$ years 7 months, range $=5$ years 0 months to 5 years 11 months) took part ${ }^{1}$. The children were attending mainstream schools in the Stirlingshire area. They were recruited following the acquisition of ethical, education authority, school, and parental permissions. Data from these children are reported by Doherty-Sneddon \& Phelps (2005) and Phelps et al. (in press), respectively. These earlier studies looked at proportion of time spent using GA whilst answering questions. In the current investigation we looked at direction of GA whilst answering questions. By chance all the children volunteering to take part were right handed.

\section{Stimuli}

Two different types of questions were posed to the children: verbal reasoning and mental arithmetic. Both the verbal questions and the arithmetic questions were based on stimuli described in the Wechsler Preschool and Primary Scale of Intelligence (WPPSI, Wechsler, 1967). Verbal reasoning questions required the children to define words, spell words, repeat word lists, and give information about concepts. The mental arithmetic questions involved addition, subtraction, multiplication/division, and number use. The 5-year-old children answered 24 questions of each type, whereas the 8-year-old children answered 36 questions of each type. All current data is normalised by number of questions to allow for this variability. Furthermore an analysis of the order of presentation of the questions confirmed that GA frequency and direction did not change in any way between questions occurring early or later in the question. So answering more or fewer questions did not influence children's GA.

For each type of question there were two levels of difficulty: easy and hard. For the 8-year-old children, examples of easy and hard verbal questions were: 'What is a photograph'?; 'What is humour'?, respectively. Examples of easy and hard arithmetic questions were: '9-6=_’?; '265-34=_'?, respectively. For the 5-year-old children, examples of easy and hard verbal questions were: 'What is a dog'?; 'What is a telescope'?, respectively. Examples of easy and hard arithmetic questions were: ' $1+1=$ '?; ' $4+4=$ ' ?, respectively. For each age group, class teachers confirmed that half of the questions used were easy (predicted $80 \%-100 \%$ accuracy), and half moderately difficult (predicted 
$30 \%-50 \%$ accuracy). In addition, analyses of the accuracy of responses confirmed that the easy questions were answered significantly more accurately than the hard questions for both age groups (8-year-olds: mean easy $=$ $89.5 \%$; mean hard $=31.2 \%$; 5-year-olds: mean easy accuracy $=79.8 \%$, mean hard $=48.8 \%$ ). (For full details see Doherty-Sneddon \& Phelps, 2005; Phelps et al., in press).

\section{Procedure}

Children were asked the questions individually in a quiet location separate from their classroom. The ordering of question type, question difficulty, and the individual questions was fully counterbalanced across participants.

Questioning took between 15-30 minutes for each child. Children were seated directly facing the questioner across a table and were therefore able to see the face and upper body of the questioner. Since the children's data is drawn from two separate studies two different questioners were involved. However the protocols were standardised and they followed the same instructions to enable similar interactions to occur between themselves and the children. For example they both mantained gaze at the child's face during questioning. To enable quantification of children's direction of eye gaze during the thinking stage of the question-answer interaction, a front-on view of each child's head and shoulders was video-recorded throughout testing using a digital camcorder. Whilst all children were fully aware that they were being filmed, they were not aware that their gaze behaviour was of specific interest.

Two coders (blind to the experimental hypotheses relating age and question difficulty to direction of GA) scored the direction of each child's GA during thinking (i.e., the interval between the questioner having finished speaking and the child having started speaking) for each question, for which there was $100 \%$ agreement. In earlier studies we have documented patterns of GA occurrence also while children listen to questions and speak their response (Doherty-Sneddon et al 2002). During these phases of an interaction GA is typically very low especially for young children (e.g. only $13 \%$ of speaking time is associated with GA when 5-year-olds are answering arithmetic questions, see Doherty-Sneddon et al., 2002). For this reason we have in recent studies, including the current, focused our attention on the thinking period only as this is the part of interactions most associated with GA in response to cognitive difficulty. There were nine different directions of GA movements: Right-down, righthorizontal, right-up, left-down, left-horizontal, left-up, vertical-up, vertical-down, and flicks, which comprised rapid eye movements in a sequence of 2 or more directions, each of which lasted $500 \mathrm{~ms}$ or less. There was also the

\footnotetext{
${ }^{1}$ Gender did not have any effect on the GA behaviours under investigation nor interact with any of the other independent
} 
additional constraint that the first gaze direction of this sequence differ to that of the immediately preceding listening stage. The $500 \mathrm{~ms}$ cutoff was chosen as movements less than this could reflect purely saccadic movements (Schiller, Sadbell \& Maunsell, 1987). The mean number of movements per thinking episode was 2.72. If multiple directions of sustained (more than 500ms) GA movements (i.e., all categories except flicks) occurred during a given thinking episode, each GA direction was scored as having occurred. The dependent variable was the number of GA movements per question (dependant variable = total GA movements of each type / number of questions in the condition).

\section{Results}

\section{Profiles of GA direction}

The mean number of GA movements in each Direction per question, split by Age of child, Question Type, and Question Difficulty, are shown in Table 1. As can be seen, differences between age groups in patterns of direction of GA during thought are evident. The 5-year-old children engaged in a high number of flicks (rapid eye movements in multiple directions) with comparatively less sustained gaze shifts in any particular direction. In contrast the 8-year old children hardly ever engaged in flicks, and instead most frequently averted their gaze to the right (up and down) and to the left (up). In addition Table 1 shows that more instances of GA movements occurred when the 8-year-old children answered arithmetic questions compared with verbal questions; and when both groups of children answered hard questions compared with easy questions.

A 4-way ANOVA was used, with Direction of GA (9 levels: right-down, right-horizontal, right-up, left-down, lefthorizontal, left-up, vertical-up, vertical-down, flitting gaze), Question Type (2 levels: verbal, arithmetic), and Question Difficulty (2 levels: easy, hard) acting as within subjects variables and Age (2 levels: 5 years; 8 years) acting as a between subjects variable. The number of GA movements per question was the dependent variable.

All main effects proved significant: Direction of $\mathrm{GA} \underline{\mathrm{F}}(8,280)=2.04, \eta_{\mathrm{p}}{ }^{2}=.06, \underline{\mathrm{p}}<.05$, with the most prevalent gaze direction types used being flicks, up right and down right movements (see Table 1); Age $\underline{\mathrm{F}}(1,35)=10.54, \eta_{\mathrm{p}}{ }^{2}=.23$ $\mathrm{p}<.001$, with the 8-year old children using more movements overall, (mean number of movements per question: 5 year-olds $=.09(\mathrm{SD} .04) ; 8$-year-olds $=.14(\mathrm{SD} .05))$; Question Type, $\underline{\mathrm{F}}(1,35)=21.72, \eta_{\mathrm{p}}{ }^{2}=.38, \underline{\mathrm{p}}<.001$, with 
more GA movements to arithmetic questions, (mean number of movements: verbal $=.10(\mathrm{SD} .04) ;$ arithmetic $=.14$ (SD .09)); and Question Difficulty, $\underline{\mathrm{F}}(1,35)=67.49, \eta_{\mathrm{p}}{ }^{2}=.66, \underline{\mathrm{p}}<.001$, with more GA movements to hard questions, (mean number of movements per question: easy $=.08(\mathrm{SD} .03)$; hard $=.16(\mathrm{SD} .08))$.

Direction of GA was found to interact significantly with all of the other variables: Age, $\underline{\mathrm{F}}(8,280)=19.26, \eta_{\mathrm{p}}{ }^{2}=.36$, $\underline{\mathrm{p}}<.001$; Question Difficulty, $\underline{\mathrm{F}}(8,280)=4.69, \eta_{\mathrm{p}}{ }^{2}=.12, \underline{\mathrm{p}}<.001$; and Question Type, $\underline{\mathrm{F}}(8,280)=3.64, \eta_{\mathrm{p}}{ }^{2}=.08, \underline{\mathrm{p}}$ $<.01$ (see Table 1 for means). Likewise, Question Type was also found to interact significantly with all of the other variables: Direction of GA, $\underline{\mathrm{F}}(8,280)=3.64, \eta_{\mathrm{p}}{ }^{2}=.08, \underline{\mathrm{p}}<.01$ (as above); Age, $\underline{\mathrm{F}}(1,35)=45.11, \eta_{\mathrm{p}}{ }^{2}=.56, \underline{\mathrm{p}}<.001$; and Difficulty $\underline{\mathrm{F}}(1,35)=15.55, \eta_{\mathrm{p}}{ }^{2}=.31, \underline{\mathrm{p}}<.001$. In addition Age and Question Difficulty interacted, $\underline{\mathrm{F}}(1,35)=$ $28.46, \eta_{\mathrm{p}}{ }^{2}=.45, \underline{\mathrm{p}}<.001$. All of these 2 -way interactions were further qualified by two significant 3 -way interactions, as described below.

Left hemisphere tasks relate to rightward movements? Post-hoc t-tests, with Bonferroni adjustments for multiple comparisons (2-tailed), revealed that for the 8-year-olds right up movements were significantly more prevalent than left lateral, flick, right lateral, and vertical up $(\underline{t}(18)=4.55, \underline{\mathrm{p}}<.01 ; \underline{\mathrm{t}}(18)=6.0, \underline{\mathrm{p}}<.001 ; \underline{\mathrm{t}}(18)=$ $4.91, \underline{\mathrm{p}}<.01) ; \underline{\mathrm{t}}(18)=4.26, \underline{\mathrm{p}}<.02$ respectively). Finally right down movements occurred significantly more often than left lateral, flick, right lateral, vertical up movements $(\underline{\mathrm{t}}(18)=5.38, \underline{\mathrm{p}}<.001 ; \underline{\mathrm{t}}(18)=8.23, \underline{\mathrm{p}}<.001 ; \underline{\mathrm{t}}(18)=$ $6.20, \underline{\mathrm{p}}<.001 ; \underline{\mathrm{t}}(18)=5.0, \underline{\mathrm{p}}<.01$, respectively). These results show right up and right down movements to be the most prevalent movements of the 8-year-old children. An overall leftward movement score was calculated for the older children (i.e. the mean of the left up, left down and left lateral scores) and compared with the overall rightward movement score. A t-test showed that 8-year-old children used significantly more rightward movements overall than leftward movements, $\underline{\mathrm{t}}(18)=2.22, \underline{\mathrm{p}}<.05$ (mean leftward $=.13$, SD .14; mean right $=.23$, SD .12$)$. A similar overall up versus down analysis showed no difference, $\underline{t}(18)=1.19, \underline{p}=.25$ (mean up $=.21$, SD .12; mean down $=.17$, SD .08).

Bonferroni adjusted post-hoc t-tests on the 5-year-old's data revealed that the younger children used significantly more flicks than 5 of the sustained GA directions (left down: $\underline{\mathrm{t}}(17)=7.27, \underline{\mathrm{p}}<.001$; left up: $\underline{\mathrm{t}}(17)=6.95, \mathrm{p}<.001$; right up: $\underline{\mathrm{t}}(17)=7.76, \underline{\mathrm{p}}<.001$; right lateral: $\underline{\mathrm{t}}(17)=4.44, \underline{\mathrm{p}}<.05$; right down: $\underline{\mathrm{t}}(17)=7.78, \underline{\mathrm{p}}<.001)$, means in 
Table 1. They also used significantly more left lateral movements than: left up, left down, right up and right down $(\underline{\mathrm{t}}(17)=5.58, \underline{\mathrm{p}}<.001 ; \underline{\mathrm{t}}(17)=5.63, \underline{\mathrm{p}}<.001 ; \underline{\mathrm{t}}(17)=5.78, \underline{\mathrm{p}}<.001 ;$ and $\underline{\mathrm{t}}(17)=6.23, \underline{\mathrm{p}}<.05$ respectively)

GA changes with age? The interaction between Age and Question difficulty reported above showed that both ages groups increased the number of GA movements made when questions got harder (5-year-olds, $\underline{t}(17)=3.82, \underline{p}<.01$, mean easy $=.08(\mathrm{SD} .03)$, mean hard $=.11(\mathrm{SD} .05) ; 8$-year-olds, $\underline{\mathrm{t}}(18)=7.50, \underline{\mathrm{p}}<.001$, mean easy $=.08(\mathrm{SD} .03)$, mean hard $=.21(\mathrm{SD} .08))$. While both ages used equivalent numbers of movements during easy questions, $\mathrm{t}(35)=$ $.09, \underline{p}=.93$, the older children used more movements than the younger during difficult questions, $\underline{t}(35)=4.47, \underline{p}<$ .001. This may reflect the fact that the harder questions were marginally more challenging for the older children (see methods section for reporting of mean accuracy scores). Bonferroni adjusted post-hoc t-tests across the 2 age groups revealed that the 5-year-olds used significantly more flicks and left lateral movements compared with the older children $(\underline{\mathrm{t}}(35)=8.10, \underline{\mathrm{p}}<.001 ;$ and $\underline{\mathrm{t}}(35)=3.59, \underline{\mathrm{p}}<.01$, respectively). In contrast the 8-year-old children used significantly more left up, left down, right up and right down movements than the younger children $(\mathrm{t}(35)=$ $2.95, \mathrm{p}<.01 ; \underline{\mathrm{t}}(35)=2.53, \mathrm{p}<.05 ; \underline{\mathrm{t}}(35)=5.78, \mathrm{p}<.001 ; \underline{\mathrm{t}}(35)=7.72, \mathrm{p}<.001$, respectively). Means in Table 1 . While both groups of children therefore increased their gaze aversion movements in response to increasing question difficulty the increases reflected the prevalent movement types of each age group as shown by the 3-way interaction between Direction of GA, Age and Question Difficulty as described below, with the profile of GA directions remaining the same regardless of Question Difficulty. Any difference in how challenging questions were in the two groups cannot therefore be the source of difference in GA directions between the two age groups.

Cognitive difficulty and direction of GA? There was a significant 3-way interaction between Direction of GA, Age, and Question Difficulty, $\underline{\mathrm{F}}(8,280)=11.37, \eta_{\mathrm{p}}{ }^{2}=.25, \underline{\mathrm{p}}<.001$ (see Table 1 for means). Simple main effects showed that the 8-year-old children increased the number of GA movements when answering difficult questions compared with easy questions for all directions of GA except flicks (left up $\underline{F}(1,18)=8.87, \eta_{\mathrm{p}}{ }^{2}=.33, \underline{p}<.01$; left lateral $\underline{\mathrm{F}}(1,18)=5.54, \eta_{\mathrm{p}}{ }^{2}=.03, \underline{\mathrm{p}}<.05$; left down $\underline{\mathrm{F}}(1,18)=14.28, \eta_{\mathrm{p}}{ }^{2}=.44, \underline{\mathrm{p}}<.001 ;$ right up $\underline{\mathrm{F}}(1,18)=32.45, \eta_{\mathrm{p}}{ }^{2}$ $=.64, \underline{\mathrm{p}}<.001 ;$ right lateral $\underline{\mathrm{F}}(1,18)=9.20, \eta_{\mathrm{p}}{ }^{2}=.34, \underline{\mathrm{p}}<.01 ;$ right down $\underline{\mathrm{F}}(1,18)=70.10, \eta_{\mathrm{p}}{ }^{2}=.80, \mathrm{p}<.001 ;$ vertical up $\underline{\mathrm{F}}(1,18),=15.45, \eta_{\mathrm{p}}{ }^{2}=.46, \underline{\mathrm{p}}<.001 ;$ vertical down $\underline{\mathrm{F}}(1,18)=16.28, \eta_{\mathrm{p}}{ }^{2}=.48, \underline{\mathrm{p}}<.01$. In contrast the 5year-old children increased their use of flicks as questions got harder, $\underline{\mathrm{F}}(1,17)=30.16, \eta_{\mathrm{p}}{ }^{2}=.64, \underline{\mathrm{p}}<.001$ (see 
Table 1). So for both age groups the profiles of GA direction remained the same with 'more of the same' occurring during difficult questions.

Finally there was a significant 3-way interaction between Question Type, Age and Question Difficulty, $\underline{\mathrm{F}}(1,35)=$ $16.27, \eta_{\mathrm{p}}{ }^{2}=.32, \mathrm{p}<.001$. Post-hoc t-tests revealed that maths questions elicited more GA movements than verbal questions for both easy and hard trials for 8 -year-olds $(\underline{\mathrm{t}}(18)=3.73, \underline{\mathrm{p}}<.01 ; \underline{\mathrm{t}}(18)=5.57, \underline{\mathrm{p}}<.001$ respectively). In contrast verbal questions elicited a greater number of movements for the younger children on both easy and difficult trials $(\underline{\mathrm{t}}(17)=2.47, \underline{\mathrm{p}}<.05 ; \underline{\mathrm{t}}(17)=2.70, \underline{\mathrm{p}}<.05$, respectively $)$.

As described above, 5-year-old children mainly use flicks during GA with some use of sustained left lateral movements. An analysis of the individual propensities to use particular gaze directions showed that the majority of 8-year-old children (68.4\%) predominantly used sustained rightward movements, thus providing some support for the idea that these older children were using contralateral LEMs. Although this directional preference was not true of all the 8 -year old children $(26.3 \%=$ predominantly 'left movers'; $5.3 \%=$ no predominant direction of GA $)$. A similar analysis on the 5-year-olds' data showed that $72 \%$ of them primarily used flicks, while $11 \%$ primarily used sustained left lateral movements. The remaining $17 \%$ were equally divided between right lateral, vertical up and vertical down movers.

In summary direction of GA was shown to be influenced by the age of thinker. So, as can be seen in Table 1, the 5year-old children predominantly used flicks, with comparatively little use of other (sustained) directions of GA, although they also used sustained left lateral movements. In contrast, the 8-year-old children predominantly used sustained right down, right up, and left up movements with very few flicks. Neither question type nor question difficulty influenced the profile of directions of GA used so, for example the younger children primarily used flicks and left laterals regardless of whether they were answering arithmetic or verbal questions, easy or hard questions. However both question type and difficulty influenced the number of GA movements occurring. So, there were more GA movements to hard questions than easy questions but no evidence of a particular shift to, for example more up movements with increasing cognitive difficulty (cf. Previc et al 2005).

\section{Discussion}


The current research looked at 5- and 8-year-old children's patterns of GA movements away from the face of an adult questioner whilst thinking about their answers to mental arithmetic and verbal reasoning questions - two lefthemisphere tasks (Buchel, 2003; Delazer et al., 2003).

\section{Patterns of GA movements across groups}

Previous research has identified developmental differences between 5- and 8-year-old children in terms of the proportion of time spent in GA during thought. So, 8-year-old children use higher proportions of GA per se than 5year-old children. Further, whilst both age groups increase their use of GA in response to increasing task difficulty, 8-year-old children do so in a more consistent manner than 5-year-old children (Doherty-Sneddon et al., 2002).

Consistent with those studies which identify developmental trajectories in the proportion of time children spend using GA during thought (e.g. Doherty-Sneddon et al., 2002; Phelps et al., in press), the current study has further identified another aspect of GA which differs across these two age groups. Not only did the older children engage in more movements overall than the younger children, they predominantly used sustained rightward movements (both up and down) while the 5-year-olds predominantly used flicks (rapid multi-directional movements). Changes in general amounts of face gaze with age are reported in the literature. For example Doherty-Sneddon (1995) reports a tendency for younger children to look at one another more than older children and adults during collaborative tasks.

While the age groups use different styles of GA they may serve the same function- to switch off from the face of the questioner. Evidence for this is that: like the sustained movements of older children, younger children's GA also primarily occurs during the thinking phases of the interactions with very little during speaking or listening (e.g. $13 \%$ of speaking time, Doherty-Sneddon et al 2002). In addition the number of flicks increases with question difficulty. These are the same patterns of occurrence observed in the sustained GA movements of older children. Furthermore although we cannot directly test any benefit to performance of GA in the current data (because GA increases with increased question difficulty- and hence reduced performance), we have shown significant performance benefits when 5-year olds are encouraged to increase their GA (Phelps et al, in press) showing that GA produced by children of this age carries a functional benefit to problem solving in the same way that it does for older participants (Glenberg et al 1998). However it is not clear whether 'trained' GA in the Phelps et al paper is 
the same as spontaneous GA in this age group. For example the GA primarily consisted of sustained movements (Phelps, personal communication) which makes sense given that the children were making a deliberate attempt to look away from the questioner's face as instructed. It may be that flicks do not provide the functional benefit that sustained aversions do. The number of movements could be an indicator of task difficulty without funcitonally interacting with problem solving behaviour, because simply initiating the aversion may not be enough to get a benefit from it. This is consonant with the attention shifting model put forward in this paper. A low level attention shifting mechanism is activated when task demands are high (a possible neurological substrate might be the superior colliculus) this initiates automatic saccade-like movements that allow some disengagement from the IPS. The use of uncoordinated rapid flicking movements suggests that, for these younger children, GA represents an 'unfocused'/diffuse blocking of the external environment. Such unfocused behaviours parallel the sorts of selfstimulatory and fidgeting behaviours sometimes observed when young children are concentrating (e.g. DohertySneddon, 2003). More sustained aversions are possible with increased development as executive control over attention shifting improves (possibly mediated by activity in the prefrontal cortex) that allow a functional benefit of GA in older children. Neuroimaging studies of activation during GA would be invaluable in helping us understand the development of these behaviours.

The 8-year-old children's predominant use of rightward GA movements likely reflects the fact that they are engaging in contralateral eye movements in response to the two left-hemisphere tasks (Buchel, 2003; Delazer et al., 2003). In contrast to Previc et al's (2005) results with 11-year-old children, the current 8-year-olds did not show an upward bias. One reason for the difference in findings might be that the Previc et al 11-year-olds used more abstract reasoning than our 8-year-olds simply because they were older. In addition there were important methodological differences between the studies. For example the experimenter was behind the participants during questioning in the Previc study whereas our testing was done face-to-face, a factor that has been shown to influence the direction of GA movements (e.g. Gur et al, 1975). Furthermore in our current study participants answered arithmetic and verbal questions (such as vocabulary) all involving fairly concrete problem solving. Participants in the Previc study answered questions designed to elicit abstract reasoning - explaining the meaning behind proverbs - and completed items involving visuospatial problem solving. 
The younger children primarily used flicks but did also engage in a significant number of sustained left lateral movements. This is difficult to explain given that the verbal and arithmetic tasks should already be lateralised in this age group (e.g. Mills, Coffey-Corina, \& Neville, 1997). However left lateral movements do not increase with task difficulty in the way that flicks do for these children suggesting that they are not associated with attentional resources in the way that the sustained movements of the older children are. Furthermore Gur et al (1975) propose that the additional cognitive burden of anxiety during face-to-face questioning can cause participants to default to their dominant hemisphere, irrespective of whether this represents the 'best' hemisphere for the problem type. Given that all the children were right handed, the pattern of results suggests that the younger children did indeed rely on their right hemisphere for task processing in the current situation - hence the left-ward movements observed. All the children were right handed in the current study by chance and future research with left handed children would illuminate whether the directional patterns of both the younger and older children reported here are influenced by handedness.

There are clearly important educational implications for this research. Previous research has shown that teachers and other professionals working with and assessing children could potentially use certain patterns of gaze and GA as an indications of thinking and cognitive effort (Doherty-Sneddon et al., 2002). Specifically, it has been shown that task engagement and effort are indicated by high proportions of time spent averting gaze while children think about their responses (Doherty-Sneddon et al., 2002; Phelps et al, in press; Doherty-Sneddon et al 2005). The current results extend this finding and show that the nature of GA during thinking also changes in important qualitative ways during the early primary school years with fewer flicks and more definitive movements, potentially indicating increased cognitive development. In addition the current study points to the possibility that (particularly for the younger children) the number of GA movements may well offer a more reliable cue of thought and concentration than the proportion of GA used.

A recent study by the authors shows the potential of GA as a cue in the classroom. Doherty-Sneddon \& Phelps (2005) found that in real-time classroom teaching, children's GA cues typically did not influence whether or not their teacher interrupted them either prior to or during a response they were making to a question asked of themand indeed teachers typically interrupted the child's thinking time after about 1 second regardless of the child's behaviour. While this might suggest that teachers do not use gaze cues to monitor children's comprehension or 
need for assistance, the study also showed that when asked to comment on video recordings of their interactions with their pupils teachers often referred to individual children's mental and attentional states, and these references were often linked explicitly to children's gaze behaviours. Given that teachers can indeed detect children's gaze related cues, it may be that teachers could be encouraged to develop their teaching strategies ensuring that they make full use of their intuitions about children's gaze behaviour in real-time classroom teaching. For example if a child is asked a question but doesn't respond immediately, a relatively high proportion of time spent averting gaze typically indicates that they are still thinking about what their response will be (i.e. they have not simply given up). It is therefore worthwhile waiting a little longer before interrupting to see what they child has to say. The importance of correctly identifying 'thinking time' during teacher assessments of children is noted by Davenport (2003). The current study shows that both the spatial and temporal nature of GA behaviours may vary between younger and older primary school aged children. During training seminars given by the authors' research team, teachers readily identify with GA as a good external cue of thinking. It is yet to be revealed by future training studies whether they can successfully implement this.

In conclusion, direction of GA promises to give important insights into the underlying cognitive processing of tasks by children. In younger children cognitive difficulty is reflected in the number of GA movements they make and these are predominantly rapid movements of the eyes in a number of directions. In older children the proportion of time spent averting gaze is also a useful indicator of difficulty of material they are processing and movements are predominantly in upward left and right or downward right directions possibly reflecting the nature of underlying task processing. GA in response to mental demands develops across the early primary years. The current evidence suggests the possibility that this is associated with developing attention shifting mechanisms although this is an issue to be directly addressed in future research. 


\section{References}

Bakan, P. (1969). Hypnotizability, Laterality of Eye Movements and Functional Brain Asymmetry. Perceptual and Motor Skills, 28, 927-932.

Banich, M.T. (2004). Cognitive Neuroscience and Neuropsychology. Houghton Mifflin: Boston.

Beattie, G.W. (1978). Floor Apportionment and Gaze in Conversational Dyads. British Journal of Social and Clinical Psychology, 17, 7-16.

Beattie, G. W. (1981). A further investigation of the cognitive interference hypothesis of gaze patterns during conversation. British Journal of Social Psychology, 20, 243-248.

Buchel, C. (2003). Cortical hierarchy turned on its head. Nature Neuroscience, 6, 657-658.

Davenport, N.A.M. (2003). Questions, Answers and Wait-time: Implications for assessment of young children. International Journal of Early Years Education, 11, 245-253.

Day, M. E (1964). An Eye Movement Phenomenon Relating to Attention, Thought and Anxiety. Perceptual and Motor Skills, 19, 443-446.

Delazer, M., Domahs, F., Bartha, L., Brenneis, C., Lochy, A., Trieb, T., \& Benke, T., (2003). Learning complex arithmetic-an fMRI study. Cognitive Brain Research, 18, 76-88.

Doherty-Sneddon, G. (2003). Children's Unspoken Language. Jessica Kingsley Publishers, London.

Doherty-Sneddon, G., Bruce, V. Bonner, L., Longbotham, S. \& Doyle, C. (2002). Development of Gaze Aversion as Disengagement from Visual Information. Developmental Psychology, 38, 438-445.

Doherty-Sneddon, G., \& Phelps, F.G. (2005). Gaze aversion: A response to cognitive or social difficulty? Memory \& Cognition, 33(4), 727-733.

Duke, J. D. (1968). Lateral Eye Movement Behavior. The Journal of General Psychology, 78, 189-195.

Galluscio, E.H. \& Paradzinski, P. (1995). Task specific conjugate lateral eye movements. Perceptual and Motor Skills, 81, 755-762.

Glenberg, A. M. (1997). What memory is for. Behavioural and Brain Sciences, 20, 1-19.

Glenberg, A. M., Schroeder, J. L., \& Robertson, D. A. (1998). Averting the gaze disengages the environment and facilitates remembering. Memory and Cognition, 26, 651-658.

Griffiths, P. \& Woodman, C. (1985). Conjugate Lateral Eye Movements and Cognitive Mode: Blindness as a Control fort Visually- Induces Oculomotor Effects. Neuropsychologia, 23 (2), 257-262. 
Gur, R. E, Gur, R. C, and Harris, L. J. (1975). Cerebral Activation, as Measured by Subjects' Lateral Eye Movements, is Influenced by Experimenter Location. Neuropsychologia, 13, 35-44.

Kane, M.J., and Engle, R.W. (2002). The role of prefrontal cortex in working-memory capacity, executive attention, and general fluid intelligence: An individual differences perspective. Psychonomic Bulletin and Review, 9, 637-671.

Kinsbourne, M. (1972). Eye and Head Turning Indicates Cerebral Lateralization. Science, 176, 539-541.

Kocel, K., Galin, D., Ornstein, R., and Merrin, E.L. (1972). Lateral eye movement and cognitive mode. Psychonomic Science, 27, 223-224.

Mills, D.L., Coffey-Corina, S., \& Neville, H.J. (1997). Language comprehension and cerebral specialization from 13 to 20 months. Developmental Neuropsychology, 13, 397-445.

Paus, T. (1996). Location and function of the human frontal eye field: A selective review. Neuropsychologia, 34, 475-483.

Paus, T., Koski, L., Caramanos, Z., \& Westbury, C. (1998). Regional difficerences in the effects of task difficulty and motor output on blood flow responses in the human anterior cinulate cortex. A review of 107 PET activation studies, NeuroReport, 9, R37-R47.

Pearson, D.A.M., \& Lane, D.M. (1991). Auditory attention shifting: a developmental study. Journal of Experimental Child Psychology, 51, 320-334.

Phelps, F., Doherty-Sneddon, G., \& Warnock (2006). Functional benefits of children's gaze aversion during questioning. British Journal of Developmental Psychology, 24, 577-588.

Pierrot-Deseilligny, C., Muri, R.M., Ploner, C.J., Gaymard, B., \& Rivaud-Pechoux, S. (2003). Cortical control of ocular saccades in humans: A model for motricity. Progress in Brain Research, 142, 3-17.

Previc, F.H., Declerck, C., and de Brabander, B., (2005). Why your "head is in the clouds" during thinking: The relationship between cognition and upper space. Acta Psychologica, 118, 7-24.

Previc, F.H. \& Murphy, S.J. (1997). Vertical eye movements during mental tasks: A re-examination and hypothesis. Perceptual and Motor Skills, 84, 835-847.

Robertson, D. A. (1968). Eye Movement Control in Primates. Science, 161, 1219-1224.

Schiller, P.H., Sandell, J.H., \& Maunsel, J.H.R. ((1987). The effect of frontal eye field and superior colliculs lesions on saccadic latencies in the Rhesus monkey. Journal of Neurophysiology, 57, 1033-1049. 
Vrij, A. (2002). Deception in chikldren: A literature review and implications for chikldren's testimony. In H.L.

Westcott, G.M. Davies, \& R.H. C. Bull. (Eds). Children’s Testimony (pp. 175-194). London: Wiley.

Zuckerman, M. \& Driver, R.E. (1985). Telling Lies: verbal and nonverbal correlates of deception. In A.W.

Seigman \& S. Feldstein. (Eds). Multichannel integrations of nonverbal behaviour (pp. 129-147). Hillsdale: NJ:

Erlbaum. 
Table 1. Number of gaze aversion movements per question in each direction by age and question difficulty. The most frequent directions of movements for each age group are in bold type. $[\mathrm{V}=$ verbal questions; $\mathrm{A}=$ arithmetic questions].

\begin{tabular}{|c|c|c|c|c|c|c|}
\hline Age group: & 5 -year o & & & 8-year o & & \\
\hline Difficulty: & Easy Hard & Overall & Easy & Hard & Overall & Mean both ages \\
\hline Qu Type: & $\mathrm{V} \quad \mathrm{A} \quad \mathrm{V} \quad \mathrm{A}$ & & $\mathrm{V}$ A & V A & & \\
\hline left up & 02.03.02.02. & .03 & .09.12 & .16 .48 & .21 & .12 \\
\hline SD & .06.05.06. 06. & .04 & .18 .19 & .18 .68 & .26 & .21 \\
\hline left lateral & .19.17.19.17 & .18 & .02.06. & .05 .07 & .05 & .11 \\
\hline SD & .21 .14 .18 .18 & .12 & .06 .16 . & .12 .09 & .10 & .13 \\
\hline left down & .04.01.05. 05 & .04 & .04 .05 . & .08 .31 & .12 & .08 \\
\hline SD & .06.03.08.06 & .04 & .07.07. & .08 .41 & .13 & .10 \\
\hline right up & .01.01.01. 01. & .01 & .15 .25 . & .30 .60 & .33 & .17 \\
\hline SD & 05.03.03. 03. & .02 & .18 .23 . & .24 .42 & .23 & .22 \\
\hline right lateral & .07.09.08.07. & .08 & .08 .08 . & .16 .04 & .09 & .08 \\
\hline SD & .12 .16 .12 .10 & .10 & .06 .09 . & .10 .20 & .09 & .09 \\
\hline right down & .00 01.01. 01. & .01 & .12 .17 . & .26 .57 & .28 & .15 \\
\hline SD & 03.03.03.03. & .01 & .13 .16 . & .20 .32 & .15 & .17 \\
\hline vertical up & .12.07. 13. & .11 & .04 .05 . & .20 .04 & .09 & .09 \\
\hline SD & 19. 13.08. & .13 & .07.08 . & .07 .19 & .07 & .10 \\
\hline vertical down & 12. 13. 13. & .13 & .02 .12 . & .07 .23 & .10 & .11 \\
\hline SD & . 17.08 .24 & .15 & .04 .23 . & .10 .30 & .16 & .15 \\
\hline flicks & .21 .16 .40 .31 & .27 & .00 .01 . & .00 .04 & .01 & .14 \\
\hline SD & .16 .11 .21 .22 &, 14 & . 00.04 . & .02 .08 & .02 & .16 \\
\hline
\end{tabular}

